\title{
A New Car-Following Model considering Driving Characteristics and Preceding Vehicle's Acceleration
}

\author{
Yong Zhang, ${ }^{1}$ Ping Ni, ${ }^{1}$ Minwei Li, ${ }^{2}$ Hao Liu, ${ }^{2}$ and Baocai Yin ${ }^{1}$ \\ ${ }^{1}$ Beijing Advanced Innovation Center for Future Internet Technology, Beijing Key Laboratory of Multimedia \\ and Intelligent Software Technology, Faculty of Information Technology, Beijing University of Technology, \\ 100 Ping Le Yuan, Chaoyang District, Beijing 100124, China \\ ${ }^{2}$ Beijing Transportation Information Center, Tower B, Shoufa Building, No. A9, Liuliqiao South Lane, Fengtai District, \\ Beijing 100073, China \\ Correspondence should be addressed to Yong Zhang; zhangyong2010@bjut.edu.cn
}

Received 5 March 2017; Revised 16 May 2017; Accepted 9 July 2017; Published 3 October 2017

Academic Editor: David F. Llorca

Copyright (C) 2017 Yong Zhang et al. This is an open access article distributed under the Creative Commons Attribution License, which permits unrestricted use, distribution, and reproduction in any medium, provided the original work is properly cited.

\begin{abstract}
In the past decades, many improved car-following models based on the full velocity difference (FVD) model have been developed. But these models do not consider the acceleration of leading vehicle. Some of them consider individual anticipation behavior of drivers, but they either do not quantitatively determine the types of driving or artificially divide the driving types rather than deriving them from actual traffic data. In this paper, driver's driving styles are firstly categorized based on actual traffic data via data mining and clustering algorithm. Secondly, a new car-following model based on FVD model is developed, taking into account individual anticipation effects and the acceleration of leading vehicle. The effect of driving characteristics and leading vehicle's acceleration on car-following behavior is further analyzed via numerical simulation. The results show that considering the acceleration of preceding vehicle in the model improves the stability of traffic flow and different driving characteristics have different influence on the stability of traffic flow.
\end{abstract}

\section{Introduction}

With the rapid growth of the global economy and the number of vehicles, the worsening traffic congestion gradually becomes a social problem affecting the lives of residents. In order to solve a series of problems caused by traffic jams, many scholars studied the mechanism of traffic congestion in many ways. The car-following model is used to simulate the traffic flow and analyze the stability of traffic flow, which is important for analyzing the formation mechanism of traffic congestion and solving the urban traffic congestion [1]. The rise of intelligent transportation system, pilot information guidance system, autonomous intelligent cruise control system, and driverless cars further promotes the attention to the car-following behavior. Typical car-following theory studies the reaction of following car to the speed change of preceding vehicle(s) and illustrates it with a mathematical model.

In 1953, Pipes [2] first proposed the car-following model which only considered the relative velocity between the vehicles without considering the impact of the distance between vehicles and other additional information on the carfollowing behavior. Obviously, this model is seriously inconsistent with the actual situation. Bando et al. [3] proposed an optimal velocity (OV) model considering the influence of distances between vehicles on the speed of following vehicle. Helbing and Tilch [4] found that too high acceleration and unrealistic deceleration occurred in the OV model when the traffic trajectory data was used to calibrate the OV function. To overcome the deficiency of the OV model, they proposed a generalized force model (GFM). The full velocity difference (FVD) model was developed by Jiang et al. [5] with the consideration of positive and negative velocity differences. Furthermore, a number of new car-following models have been carried out based on FVD model. Tang et al. $[6,7]$ proposed a car-following model with the consideration of road conditions and vehicle communication on the basis of calibrated speed-headway function. Gong et al. [8] put forward a new full velocity difference model considering 
the asymmetry of the vehicle acceleration and deceleration process on the basis of FVD model. Zheng et al. [9] presented a new car-following model with consideration of anticipation driving behavior based on FVD model. Ge et al. [10, 11] proposed a two-velocity-difference model based on an intelligent transportation system. Ge et al. [11-13] proposed a series of improved FVD models with the consideration of various factors affecting the driver to adjust the optimal speed. Sun et al. $[14,15]$ presented a car-following model considering the effect of looking backward and multiple optimal current difference. Wang et al. [16] came up with multiple velocity difference model (MVDM) and found significant increase in traffic flow stability region of MVD model compared with FVD model. In 2015, based on optimal velocity car-following model, Ge et al. [17] proposed a new model considering traffic jerk to describe the jamming transition in traffic flow on a highway. In 2017, Song et al. [18] presented an improved carfollowing model which is based on optimal velocity model considering traffic jerk and full velocity difference.

But these models only consider the common driver's driving style without taking into account the individual driving style. In the real traffic flow, the driver's age, physical fitness, reaction sensitivity, driving skills, and other individual differences lead to different car-following behaviors [1923]. Many scholars have found that drivers in the process of following and lane changing exhibit the characteristics of delays, inaccuracies, and anticipation in the process of studying car-following model and lane-changing model [24]. Because the driver's psychological-physiological activities exhibited in the process of following are uncertain and complex, all drivers' car-following behavior and free lanechange phenomena cannot be accurately described by the same mathematical model. In recent years, the influence of driving type on traffic flow stability has been studied by many scholars. Sharma [25] analyzed the passing effect with driver anticipation effect on traffic flow and concluded that passing parameter is an important parameter which plays an important role to stabilize traffic flow. In 2016, Wen et al. [26] recently analyzed the behavior of driver characteristic on a two-lane traffic flow phenomena and confirmed that traffic jam can be suppressed efficiently by considering the driver's characteristics. To further study the driver's characteristics, Gupta and Redhu [27] proposed a modified OV model by considering the timid or aggressive features of drivers behavior on traffic flow. Peng et al. [28] considered the individual driving style based on FVD model and subjectively divided the driving types into two types: timid and aggressive; then different personal driving styles are obtained by linear combination of the two types. However, these models are qualitative analysis of driving types rather than quantitative analysis, and the types of driving are based solely on human cognition instead of starting from the actual traffic data.

To solve the above-mentioned problems in these carfollowing models, clustering analysis is introduced to obtain the driving types from real vehicle trajectory data. In addition, the acceleration information of preceding vehicle is considered in a new car-following model based on the FVD model because, in the real traffic flow, the driver tends to adjust the speed of vehicle according to the acceleration and deceleration behavior of the leading vehicle [29].

The rest of the paper is organized as follows. In Section 2 we introduce an attempt to classify driving types with real traffic data by clustering. In Section 3, a new car-following model considering the acceleration of preceding vehicle is explained. In Section 4, several features of the new model tested via numerical simulation are illustrated. Finally, a short summary is given. And the main contributions of this paper are as follows:

(i) The distribution of driving types under different road conditions is quantitatively analyzed.

(ii) The traffic parameters of different driving types are analyzed.

(iii) Based on the traffic track data, this paper further proves that different drivers do have their driving habits in the process of driving.

(iv) Based on the results of quantitative analysis, a carfollowing model considering driving type and preceding vehicle's acceleration is put forward.

\section{Driving Characteristics Classification by Clustering}

Cluster analysis is a data analysis method commonly used in the field of data mining. In this paper, we divide driving type by using clustering method, which can overcome disadvantages of car-following models that introduce the driving type based on the traditional method by only considering dominant factors. The clustering method can analyze massive amounts of traffic information effectively and deeply, moreover, it can find hidden useful knowledge in the vast amounts of traffic data.

The procedure of grouping a set of objects into some subclasses, which is composed of some similar objects, is called clustering. A cluster is a collection of data objects that are similar to one another within the same cluster and are dissimilar to the objects in other clusters. Currently, there are many mainstream methods of clustering analysis. Among them, the $k$-means algorithm based on partition, with its advantages of simple, rapid, and effective mass data processing, becomes one of the top ten classic data mining algorithms. The $k$-means are based on the basic idea of $k$ points in space as centers for clustering, classifying the objects closest to them. Through iterative method, the value of the clustering center is successively updated until the best clustering results are obtained. The famous data mining software, Waikato Environment for Knowledge Analysis (Weka), proposes the $x$-means algorithm making improvement on the $k$ means algorithm. The $x$-means algorithm can automatically determine the number of clustering centers and split them "smartly." $X$-means utilizes a Bayesian Information Criterion (BIC) in order to verify the clusters number centroids that can model the data effectively. Furthermore, $X$-means uses KDtrees data structure for speed. Lastly, the user can determine the distance function to apply, the minimum and maximum volume of clusters to weigh, and the maximum volume of 


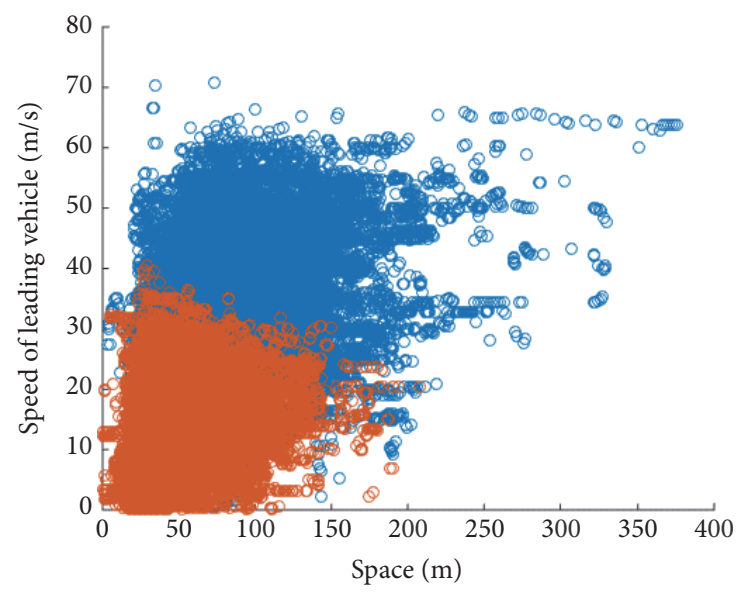

Figure 1: Clustering results of traffic states.

iterations to conduct. Initially, $X$-means algorithm starts with $K$. In this work, $K=13$ to cluster the data into thirteen clusters. First, $K$ has to be equivalent to the lower bound of the specified assortment and maintaining to add centroids where they are mandatory till the upper bound is reached. During this process, the centroid set that attains the finest score is listed, and this is one that is finally output [30]. In this article, we use the $x$-means algorithm to classify the type of driving based on the real field data.

The field data used in this paper is provided by the Federal Highway Administration's NGSIM (the Next Generation Simulation program) [31] project. Though the project was picked by the Federal Highway Administration, the features of car-following are general on the whole and the influence of nationality and region is small, so this data set has attracted the attention of scholars in the field of traffic from all countries. The data used in this study were collected on a segment of Interstate 80 in San Francisco, CA. The study segment of Interstate 80 was $1650 \mathrm{ft}$ in length and had an auxiliary lane and five freeway lanes and one on-ramp. The Interstate 80 data were collected for $45 \mathrm{~min}$ from 4:00 pm to 4:15 pm and from 5:00 pm to 5:30 pm, on April 13, 2005. Raw data was obtained frame by frame $(0.1 \mathrm{~s})$ by video processing software. To alleviate the influence of system error and testing error, in this article, a symmetric exponential moving average (SEMA) filter [32] was adopted to reduce the noise.

The trajectory data of Interstate 80 is filtered and the data without preceding car, with no front frame, or with no next frame is deleted, with the space larger than $500 \mathrm{~m}$ or with the headway more than $100 \mathrm{~s}$. The trajectory data with acceleration that is not within reasonable limits $\left(\left[-3.5 \mathrm{~m} / \mathrm{s}^{2}\right.\right.$, $\left.3 \mathrm{~m} / \mathrm{s}^{2}\right]$ ), or with the following time being less than $5 \mathrm{~s}$, which is obviously not effective record of car-following state, is further removed. Finally, we can get about 22.5 million effective data sets and each data set was obtained frame by frame $(0.1 \mathrm{~s})$ by video processing software. In order to get the driving type in similar traffic environment, we select the speed and the acceleration of the front vehicle and the distance between the vehicles as the feature vector. In this article, two kinds of road traffic states are obtained by using $x$-means algorithm. As shown in Figure 1, the traffic state represented by the blue color is significantly more unblocked than the red color. Then, we select the speed and the acceleration of subject vehicle, the speed difference, the acceleration difference, and the headway between leading vehicle and subject vehicle as the feature vector of $x$-means algorithm. Finally the trajectory data of two kinds of traffic states are clustered into three driving styles. The clustering results of the trajectory data in a unblocked traffic state are shown in Figures 2(a), 2(b), and 2(c); the results of the clustering of the trajectory data in the congested traffic state are shown in Figures 3(a), 3(b), and 3(c). It can be seen from Figure 2(b) that vehicles of the trajectory data represented by the blue color keep similar acceleration to the leading vehicles in an unblocked state of traffic, while vehicles travel at a significantly lower speed than leading vehicles' speed in the class of trajectory data represented by red color. And in the class of trajectory data represented by yellow color, the following vehicles' acceleration is significantly higher than the acceleration of the preceding vehicles. In the case of congested traffic, the speed of the vehicle is significantly lower than speed of vehicle in the unblocked traffic state. As can be seen from Figures 3(a) and 3(b), in the class of trajectory data represented by yellow color, the following vehicles' acceleration is significantly slower than the acceleration of leading vehicles and the vehicles are traveling at a slower speed than the leading vehicles in the class of trajectory data represented by blue color. Meanwhile, the speed and acceleration of the following vehicles are higher than those of the front vehicles in the class of trajectory data represented by red color. The distribution of the feature vectors of different driving types under two traffic conditions is shown in Tables 1 and 2 . In this paper, three types of driving are labeled as regular, aggressive, and conservative, respectively.

In Tables 1 and 2, we can find that type 1 corresponds to the red bubble in Figures 2 and 3, type 2 corresponds to the blue bubble, and type 3 corresponds to the yellow bubble, respectively. From Table 1, we can find that the average acceleration of vehicle, the average velocity difference, and the average acceleration difference are the dominant factors of driving types under the condition of unblocked traffic. According to these three variables, we can find that type 1 in Table 1 represents the conservative type, type 2 is the regular type, and type 3 is the aggressive type. Correspondingly, the yellow bubble in Figure 2 represents aggressive behavior, the blue bubble in Figure 2 represents the regular behavior, and the red bubble in Figure 2 represents the conservative behavior. Through the observation of Table 2, we can find that the average speed of vehicle, the average headway, and the average velocity difference are the dominant factors of driving types under the condition of congested traffic. According to these three variables, we can find that type 1 in Table 2 represents the aggressive type, type 2 is the conservative type, and type 3 is the regular type. And correspondingly, the yellow bubble in Figure 3 represents regular behavior, the blue bubble in Figure 3 represents the conservative behavior, and the red bubble in Figure 3 represents the aggressive behavior. From Tables 1 and 2, we can find that traffic data was dominated by regular driving style in the unblocked traffic state, while traffic data was dominated by aggressive 


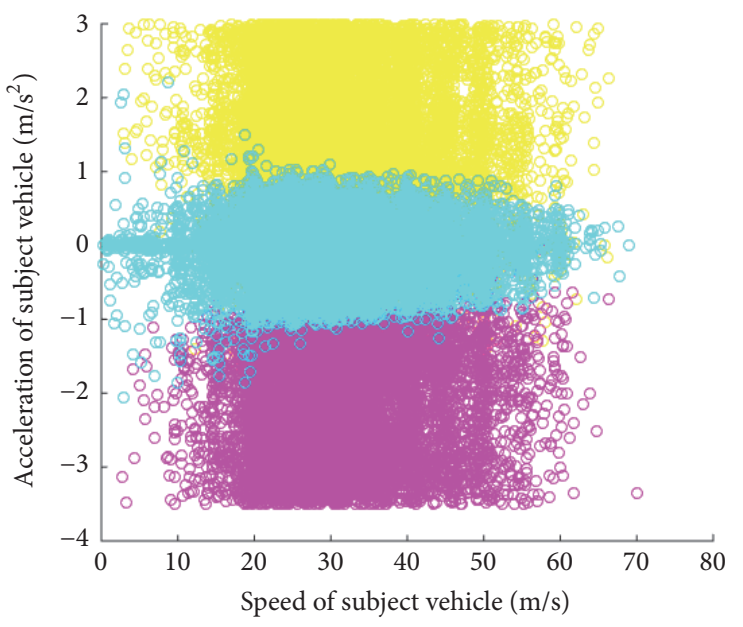

(a)

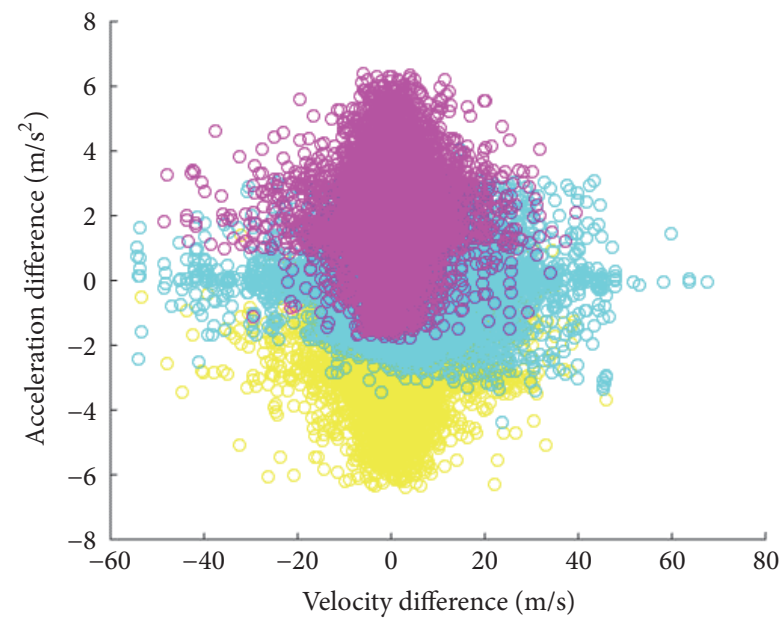

(b)

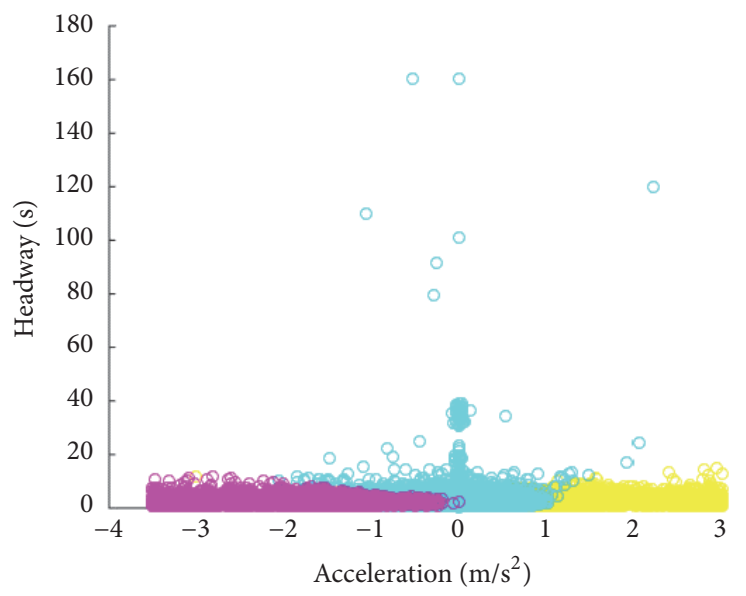

(c)

FIGURE 2: Driving types clustered under the condition of unblocked traffic.

TABLE 1: The distribution of feature vector of different driving types in an unblocked traffic state.

\begin{tabular}{|c|c|c|c|c|c|c|c|c|c|c|c|}
\hline \multirow[t]{2}{*}{ Type } & \multicolumn{2}{|c|}{$\begin{array}{l}\text { Average speed of } \\
\text { vehicle } \\
(\mathrm{m} / \mathrm{s})\end{array}$} & \multicolumn{2}{|c|}{$\begin{array}{c}\text { Average acceleration } \\
\text { of vehicle } \\
\left(\mathrm{m} / \mathrm{s}^{2}\right)\end{array}$} & \multicolumn{2}{|c|}{$\begin{array}{l}\text { Average headway } \\
\text { (s) }\end{array}$} & \multicolumn{2}{|c|}{$\begin{array}{l}\text { Average velocity } \\
\text { difference } \\
(\mathrm{m} / \mathrm{s})\end{array}$} & \multicolumn{2}{|c|}{$\begin{array}{l}\text { Average acceleration } \\
\text { difference } \\
\left(\mathrm{m} / \mathrm{s}^{2}\right)\end{array}$} & \multirow[t]{2}{*}{$\begin{array}{c}\text { Sample } \\
\text { proportion }\end{array}$} \\
\hline & Mean & STD & Mean & STD & Mean & STD & Mean & STD & Mean & STD & \\
\hline 1 & 30.5 & 8.43 & -2.09 & 0.72 & 2.42 & 1.06 & 0.19 & 5.95 & 2.14 & 1.18 & $15 \%$ \\
\hline 2 & 28.32 & 7.86 & -0.05 & 0.31 & 2.62 & 2.11 & 1.43 & 6.31 & 0.1 & 0.9 & $65 \%$ \\
\hline 3 & 30.17 & 8.4 & 1.35 & 0.88 & 2.52 & 1.37 & 0.8 & 5.94 & -2.07 & 1.15 & $20 \%$ \\
\hline
\end{tabular}

STD: standard deviation.

TABLE 2: The distribution of feature vector of different driving types in a congested traffic state.

\begin{tabular}{cccccccccccc}
\hline Type & $\begin{array}{c}\text { Average speed of } \\
\text { vehicle } \\
(\mathrm{m} / \mathrm{s})\end{array}$ & $\begin{array}{c}\text { Average acceleration } \\
\text { of vehicle } \\
\left(\mathrm{m} / \mathrm{s}^{2}\right)\end{array}$ & \multicolumn{2}{c}{$\begin{array}{c}\text { Average headway } \\
(\mathrm{s})\end{array}$} & \multicolumn{2}{c}{$\begin{array}{c}\text { Average velocity } \\
\text { difference } \\
(\mathrm{m} / \mathrm{s})\end{array}$} & \multicolumn{2}{c}{$\begin{array}{c}\text { Average acceleration } \\
\text { difference } \\
\left(\mathrm{m} / \mathrm{s}^{2}\right)\end{array}$} & $\begin{array}{c}\text { Sample } \\
\text { proportion }\end{array}$ \\
\hline 1 & 18.91 & 4.07 & -0.03 & 0.3 & 2.81 & 1.05 & -1.32 & 5.2 & 0.01 & 0.95 & $56 \%$ \\
2 & 7.98 & 3.17 & -0.03 & 0.25 & 6.91 & 10.24 & 1.97 & 4.72 & 0.05 & 0.9 & $33 \%$ \\
3 & 14.36 & 6.42 & -2.15 & 0.71 & 4.84 & 10.41 & 0.07 & 5.01 & 2.3 & 1.06 & $12 \%$ \\
\hline
\end{tabular}

STD: standard deviation. 


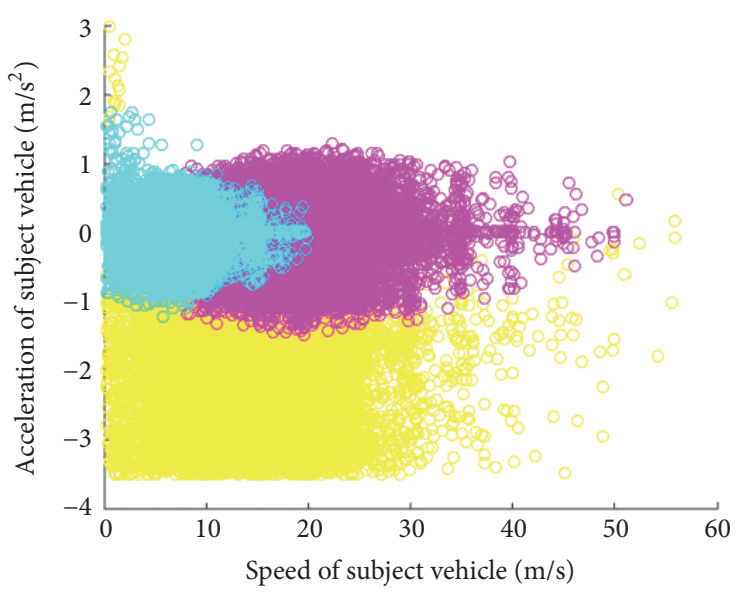

(a)

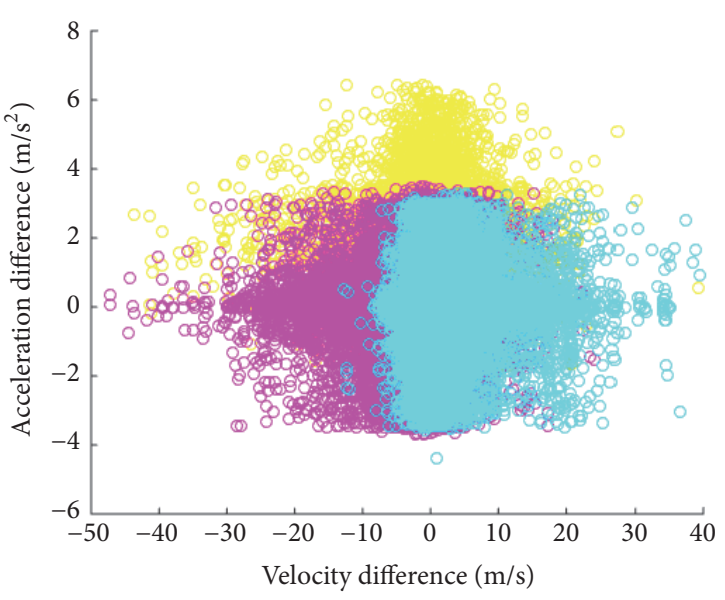

(b)

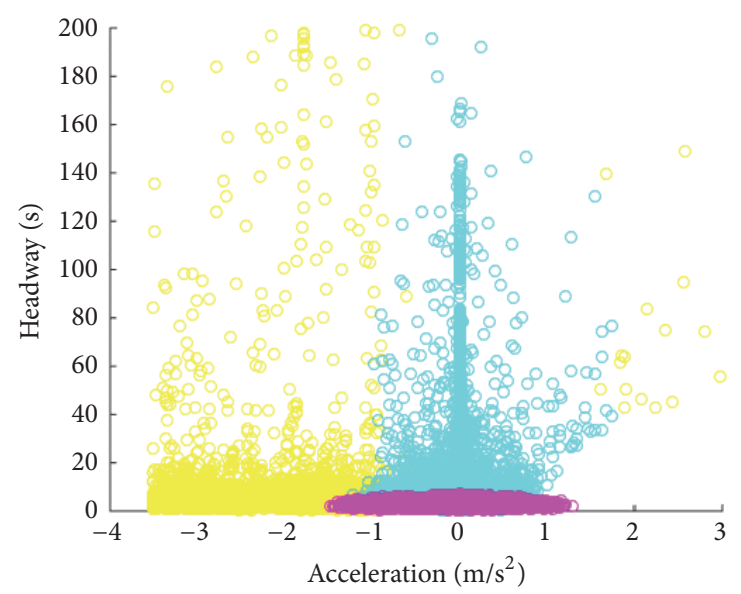

(c)

FIgURE 3: Driving types clustered under the condition of congested traffic.

driving style in the congested traffic state and the proportion of conservative driving style is also larger in the congested traffic state.

Then we take each vehicle as the research object to carry on further analysis of the driving type. We find that there are 1932 cars in the state of unblocked traffic and there are 1898 cars in the congested traffic state. Each car has a number of car-following data and each car-following data has the corresponding driving type. Then, we analyze the number of car-following data and the results of statistical analysis are shown in Figures 4(a) and 4(b). The $X$-axis of Figure 4 shows the number of vehicles, the $y$-axis represents the proportion of the dominant driving type in each vehicle's car-following data and different proportions with different colors, and the $z$-axis represents the dominant driving type of car-following data in each vehicle. As shown in Figures 4(a) and 4(b), in most cases, for the same vehicle, most of the carfollowing data (about 70\% to $90 \%$ ) are the same driving style and this proves that different drivers have different driving styles. Most traffic data belonging to cluster 1 corresponds to type 2 in Table 1 in the unblocked traffic state, and most traffic data belonging to cluster 0 corresponds to type 1 in Table 2 in the congested traffic state. This indicates that the dominant driving style is different under different road conditions.

\section{New Model}

In fact, in real traffic, due to the factors such as driving skill, age, response ability, experience, and mental and physical state, the anticipation effect of driver on the surrounding traffic environment will be different [33]. However, FVD model and most improved models based on FVD model consider the overall average response time without considering the difference of the anticipation effect caused by the driving characteristics of different drivers. In addition, most improved models on the basis of FVD model do not consider the influence of leading vehicle's acceleration on the following behavior, so they cannot describe the phenomenon that leading vehicle is accelerating and following vehicle is not slowing down when the gap between vehicles is reducing and the velocity of following vehicle is greater than preceding vehicle's [34]. For this reason, we use the $x$-means clustering algorithm to divide the driving types, and further consider the acceleration of the preceding vehicle; finally, the new model is introduced as follows: 


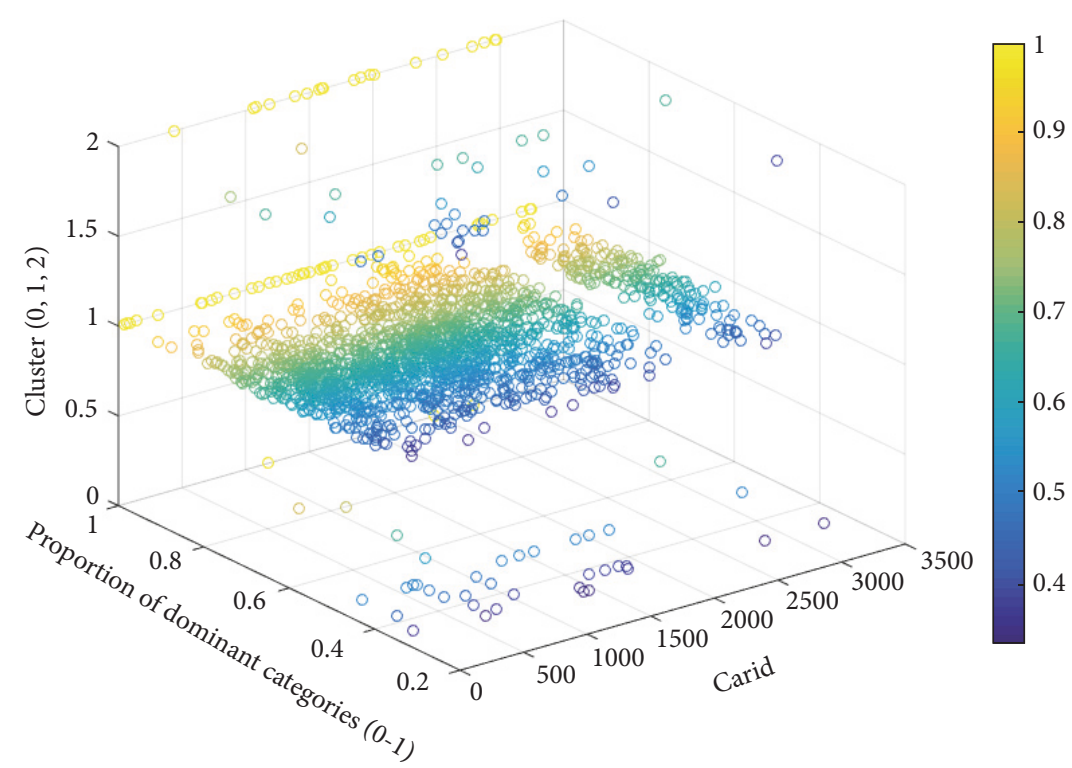

(a)

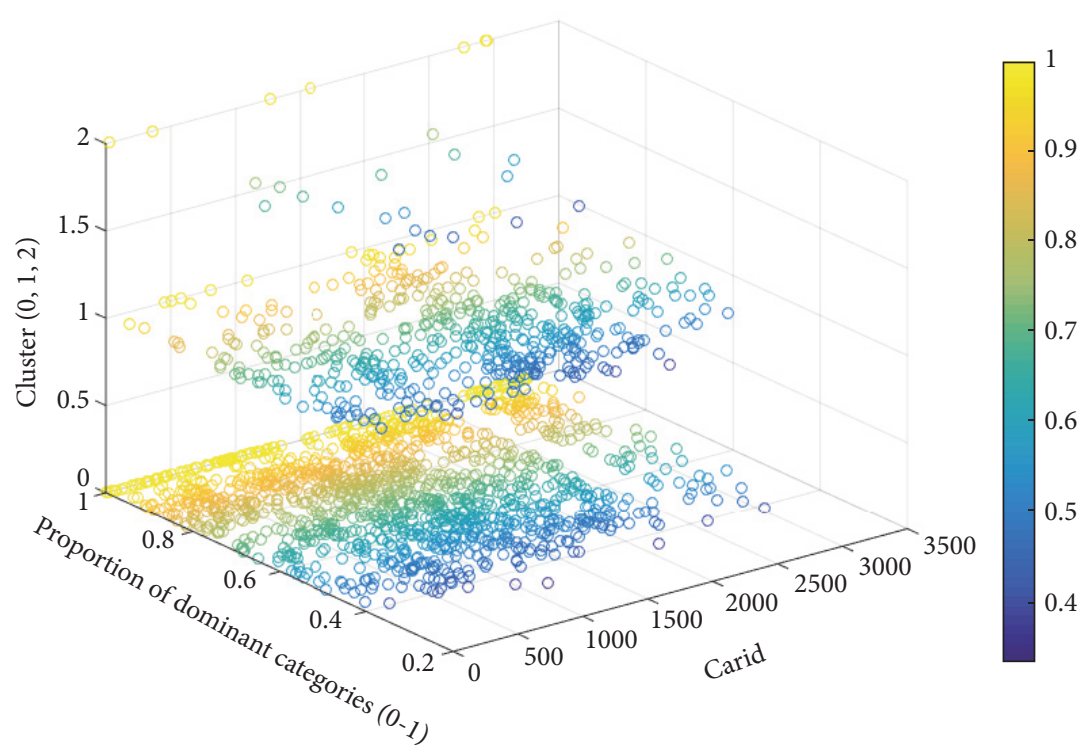

(b)

Figure 4: Statistical analysis of car-following data for each vehicle in (a) unblocked traffic state and (b) congested traffic state.

$$
\begin{aligned}
& \frac{d v_{n}(t)}{d t}=a\left[\alpha V\left(\Delta x_{n}\left(t+p_{1} \tau\right)\right)+\beta V\left(\Delta x_{n}\left(t+p_{2} \tau\right)\right)\right. \\
& \left.\quad+(1-\alpha-\beta) V\left(\Delta x_{n}\left(t+p_{3} \tau\right)\right)-v_{n}(t)\right] \\
& \quad+\lambda\left[\alpha \Delta v_{n}\left(t+q_{1} \tau\right)+\beta \Delta v_{n}\left(t+q_{2} \tau\right)\right. \\
& \left.\quad+(1-\alpha-\beta) \Delta v_{n}\left(t+q_{3} \tau\right)\right]+k a_{n+1}(t),
\end{aligned}
$$

where $0 \leq \alpha \leq 1$ and $0 \leq \beta \leq 1$ are the influence coefficients of intensity among three driving characteristics, $a$ denotes the sensitivity of the driver and is given by the reciprocal of the delay time $\tau$, namely, $a=1 / \tau, p_{1}, p_{2}, p_{3}$ are the anticipation ability coefficients response to the gap corresponding to the aggressive, regular, and conservative type of driving styles, $q_{1}, q_{2}, q_{3}$ are the anticipation ability coefficients response to the velocity difference corresponding to the three types of driving, $k$ denotes the response coefficient to the acceleration of preceding vehicle, reflecting the driver's perception in the leading vehicle's acceleration information, and $a_{n+1}(t)$ is the acceleration of car $n+1$ at time $t$. The new model degenerates into FVD model when $p_{1}=p_{2}=p_{3}=q_{1}=q_{2}=q_{3}=k=$ 0 . By expanding (1) using the first-order Taylor series, with $O\left(\tau^{2}\right)$ denoting the bounded quantity of $\tau^{2}$, the following equation can be obtained:

$$
\begin{aligned}
\Delta x_{n}(t+p \tau) & =\Delta x_{n}(t)+p \tau \frac{d \Delta x_{n}(t)}{d t}+O\left(\tau^{2}\right) \\
& =\Delta x_{n}(t)+p \tau \Delta v_{n}(t)+O\left(\tau^{2}\right)
\end{aligned}
$$




$$
\begin{aligned}
\Delta v_{n}(t+q \tau) & =\Delta v_{n}(t)+q \tau \frac{d \Delta v_{n}(t)}{d t}+O\left(\tau^{2}\right) \\
& =\Delta v_{n}(t)+q \tau \Delta a_{n}(t)+O\left(\tau^{2}\right)
\end{aligned}
$$

where $\Delta a_{n}(t)=a_{n+1}(t)-a_{n}(t)$ denotes the acceleration difference between leading car $n+1$ and following car $n$. $a_{n}(t)$ represents the acceleration of car $n$. Furthermore, (4) is obtained on the basis of (2):

$$
\begin{aligned}
V\left(\Delta x_{n}(t+p \tau)\right)= & V\left(\Delta x_{n}(t)+p \tau \Delta v_{n}(t)+O\left(\tau^{2}\right)\right) \\
= & V\left(\Delta x_{n}(t)\right) \\
& +p \tau \Delta v_{n}(t) V^{\prime}\left(\Delta x_{n}(t)\right) \\
& +O\left(\tau^{2}\right) .
\end{aligned}
$$

Therefore, we substitute (3) and (4) into (1) and obtain

$$
\begin{aligned}
& \frac{d v_{n}(t)}{d t}=a\left[V\left(\Delta x_{n}(t)\right)+\tau V^{\prime}\left(\Delta x_{n}(t)\right) \Delta v_{n}(t)\right. \\
& \left.\cdot\left(p_{1} \alpha+p_{2} \beta+(1-\alpha-\beta) p_{3}\right)-v_{n}(t)\right] \\
& +\lambda\left[\Delta v_{n}(t)+\tau \Delta a_{n}(t)\right. \\
& \left.\cdot\left(q_{1} \alpha+q_{2} \beta+(1-\alpha-\beta) q_{3}\right)\right]+k a_{n+1}(t) \\
& \quad+O\left(\tau^{2}\right) .
\end{aligned}
$$

In general, the value of the reaction time is small, and the term $O\left(\tau^{2}\right)$ in (5) is neglected because the value is relatively small in this paper. The OV function (for short, OVF) adopted in this paper is the same as FVD model [5], as shown in

$$
V(\Delta x)=V_{1}+V_{2} \tanh \left[C_{1}\left(\Delta x-l_{c}\right)-C_{2}\right],
$$

where $a=0.85 \mathrm{~s}^{-1}, V_{1}=6.75 \mathrm{~m} / \mathrm{s}, V_{2}=6.75 \mathrm{~m} / \mathrm{s}, C_{1}=$ $0.13 \mathrm{~m}^{-1}, C_{2}=1.57$, and $l_{c}=5 \mathrm{~m}$.

\section{Discussion}

4.1. Linear Stability Analysis. In order to analyze the effect of driving characteristics on jamming transition of traffic flow, linear stability analysis is carried out. Obviously, the traffic flow will be in a stable state when all vehicles run with the uniform headway $b$ and optimal velocity $V(b)$. Therefore the steady-state solution of the traffic flow is given as

$$
x_{n}^{(0)}(t)=b n+V(b) t, \quad b=\frac{L}{N},
$$

where $L$ denotes the length of road and $N$ is the total number of vehicles. A small disturbance $y_{n}(t)$ is applied to the steadystate $x_{n}^{(0)}(t)$ :

$$
y_{n}(t)=x_{n}(t)-x_{n}^{0}(t)
$$

Then substituting it into (5) and linearizing it, one can acquire results as follows:

$$
\begin{aligned}
& y_{n}^{\prime \prime}(t)=a\left[V^{\prime}(b) \Delta y_{n}(t)\right. \\
& +\tau \Delta y_{n}^{\prime}(t) V^{\prime}(b)\left(p_{1} \alpha+p_{2} \beta+(1-\alpha-\beta) p_{3}\right) \\
& \left.\quad-y_{n}^{\prime}(t)\right]+\lambda\left[\Delta y_{n}^{\prime}(t)\right. \\
& \left.\quad+\left(q_{1} \alpha+q_{2} \beta+(1-\alpha-\beta) q_{3}\right) \tau \Delta y_{n}^{\prime \prime}(t)\right] \\
& \quad+k y_{n+1}^{\prime \prime}(t),
\end{aligned}
$$

where $\Delta y_{n}(t)=y_{n+1}(t)-y_{n}(t)$ and $V^{\prime}(b)=d V\left(\Delta x_{n}\right) /$ $\left.d \Delta x_{n}\right|_{\Delta x_{n}=b}$.

Expanding $y_{n}(t)=e^{\left(i \alpha_{k} n+z t\right)}$ yields

$$
\begin{aligned}
& {\left[1-\lambda \tau\left(q_{1} \alpha+q_{2} \beta+(1-\alpha-\beta) q_{3}\right)\left(e^{i \alpha_{k}}-1\right)\right.} \\
& \left.-k e^{i \alpha_{k}}\right] \cdot z^{2}+[a \\
& -a \tau V^{\prime}(b)\left(e^{i \alpha_{k}}-1\right)\left(p_{1} \alpha+p_{2} \beta+(1-\alpha-\beta) p_{3}\right) \\
& \left.-\lambda\left(e^{i \alpha_{k}}-1\right)\right] \cdot z-a V^{\prime}(b)\left(e^{i \alpha_{k}}-1\right)=0 .
\end{aligned}
$$

Let $z=z_{1}\left(i \alpha_{k}\right)+z_{2}\left(i \alpha_{k}\right)^{2}+\cdots$ and substitute it into (10); then the first-order and second-order terms of $i \alpha_{k}$ are, respectively, deduced as follows:

$$
\begin{aligned}
& z_{1}=V^{\prime}(b) \\
& z_{2}=\frac{\left[k-1+a \tau\left(p_{1} \alpha+p_{2} \beta+(1-\alpha-\beta) p_{3}\right)\right]\left(V^{\prime}(b)\right)^{2}+(\lambda+a / 2) V^{\prime}(b)}{a} .
\end{aligned}
$$

If $z_{2}<0$, the uniform steady-state flow will be unstable, while the uniform flow is stable if $z_{2}>0$. Therefore, the neutral stability condition (i.e., $z_{2}=0$ ) is given as follows:

$$
\begin{aligned}
& V^{\prime}(b) \\
& \quad=-\frac{2 \lambda+a}{2\left[k-1+\tau\left(p_{1} \alpha+p_{2} \beta+(1-\alpha-\beta) p_{3}\right)\right]} .
\end{aligned}
$$

$$
V^{\prime}(b)<-\frac{2 \lambda+a}{2\left[k-1+\tau\left(p_{1} \alpha+p_{2} \beta+(1-\alpha-\beta) p_{3}\right)\right]}
$$

As $p_{1}=p_{2}=p_{3}=k=0$, the stable condition of the proposed model agrees with that of FVD model [5]:

$$
V^{\prime}(b)<\frac{a}{2}+\lambda
$$




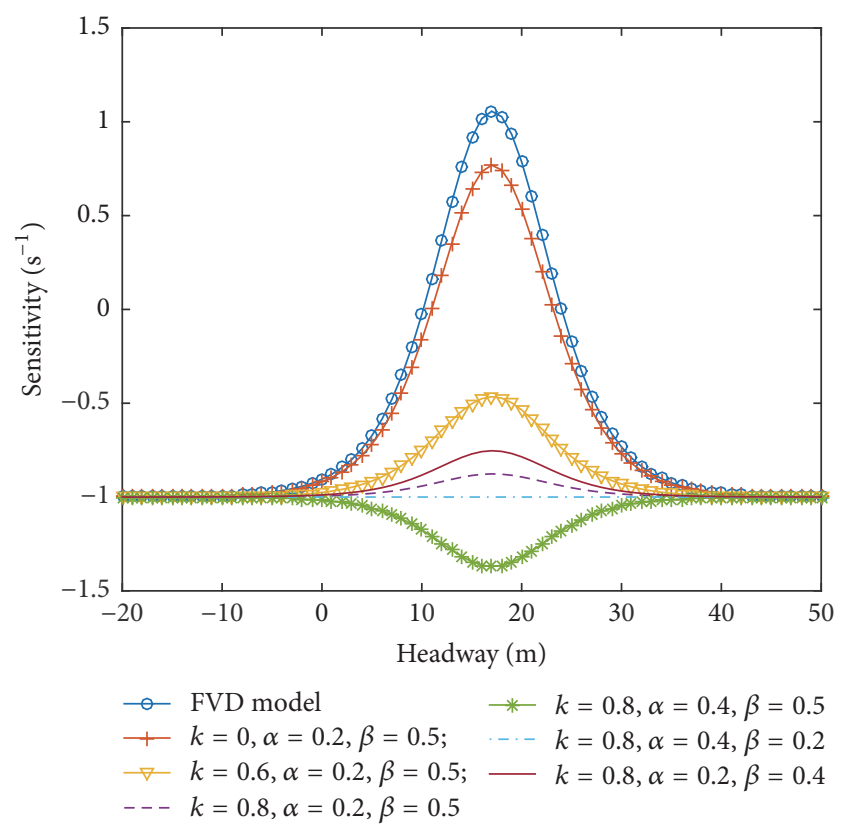

FIgURE 5: Neutral stability curves in headway-sensitivity space $(\Delta x, a)$.

According to (13), we can find that the neutral stability condition of the proposed model depends on the anticipation parameters $p_{1}, p_{2}, p_{3}$ response to the gap while having no relation with the anticipation parameters $q_{1}, q_{2}, q_{3}$ response to the velocity difference.

Figure 5 shows the neutral stable curves under different value of $k, \alpha$, and $\beta$ in the headway-sensitivity space $(\Delta x, a)$ for the proposed model with $\lambda=0.45$ and $p_{1}=0.2$, $p_{2}=0.05$, and $p_{3}=-0.2$ [28]. In Figure 5, the region above the neutral stability line means the stability region where traffic jam does not occur when small disturbances are applied. The area below the neutral stability line denotes the unstable region where the traffic flow influenced by disturbance will evolve into traffic jam with time. From Figure 5, the neutral stability curve is compressed gradually when the value of $k$ is increasing, which means that the acceleration information of preceding vehicle can improve the stability of traffic flow. Furthermore, it can be found that the neutral stability line has an apparent decline when $\alpha$ increases, which implies that the aggressive effect of driving characteristics markedly contributes to the stability of traffic flow. The neutral stability line has a certain decline when $\beta$ increases and the coefficient of $p_{3}$ decreases relatively. Moreover, the neutral stability line ascends gradually when the coefficient of $p_{3}$ is augmenting. Thus, the regular effect of driving characteristics has a certain positive role on the stability of traffic flow while the conservative effect of driving characteristics is harmful.

4.2. Starting and Braking Processes. It can be found from the analysis above that the anticipation effect of drivers has great impact on the stability of traffic flow; nevertheless, most of the car-following models up to now are built based on standard driving characteristics without considering the difference among individual drivers [33]. In fact, due to the factors such as experience, age, driving skills, response ability, and mental and physical state, the anticipation effect of drivers is different. Thus, the parameters $\alpha$ and $\beta$ are set to be random values. In order to show the impact of different anticipation effect on the traffic flow, the proposed model is used to simulate the starting and braking process with choosing the same initial condition as reference [5]. The traffic signal is red and 11 cars are waiting with the same headway of $7.4 \mathrm{~m}$ when $t<0$. Then, the traffic light is switched to green at time $t=0$, and all vehicles start to run. Other boundary conditions are set as follows: for the first car $n=11$, the optimal velocity function is $V_{11}(\infty)=14.66 \mathrm{~m} \cdot \mathrm{s}^{-1}$, and for the following car, there are $V_{n}(7.4)=0(n=1, \ldots, 10)$. For comparison, the same parameters are set as those in FVD model. Particularly, in the study of this paper, we assume that drivers have the same sensitivity to vehicle distance and speed difference information, so we make $p_{1}=q_{1}, p_{2}=q_{2}$, $p_{3}=q_{3}$.

Figure 6 depicts the simulation result of the starting process of (a) the new model and (b) FVD model. In Figure 6, parameters $\alpha$ and $\beta$ are normally distributed random variables [35] with mean value 0.3 and standard variance 0.15 . Moreover, the response coefficient to the acceleration of preceding vehicle $k$ is set as 0.02 . It can be obviously observed in (a) that the velocity curves are not uniform, which means the motion trajectories of vehicles are not all the same. The reason is that the anticipation parameters $\alpha$ and $\beta$ describing the individual difference of the driver's anticipation effect are set as random variables. This simulation result conforms to real traffic compared to that in (b). In (b), the velocity curves are very uniform and other vehicles almost all move following the pattern of the first vehicle without representing the individual difference of the drivers.

The braking process of vehicles is simulated in a similar way. The initial condition and distribution of parameters are set to be the same as those of starting process; the only difference is that the first vehicle is supposed to stop at the location of $800 \mathrm{~m}$ for a brake. Figure 7 shows the velocity curves of (a) the proposed model and (b) FVD model. The velocity curves in (a) are not uniform due to the fact that anticipation parameters of individual driver are different. But the velocity curves shown in (b) are almost all the same and, thus, are not consistent with real traffic.

The simulations of starting and braking processes indicate that our model can effectively describe the anticipation characteristic of individual driver. And given that the values of the anticipation parameters $\alpha$ and $\beta$ depend on many factors such as the age, the size of vehicles, physical fitness level of drivers, experience, and the environment of road, the exact distribution of the anticipation parameters and relevant analysis are worth more discussion in future research.

4.3. Numerical Simulation. Since the vehicle behaviors are prevalent such as lane changing, lane merging, and braking, many small disturbances can be observed in real traffic. 


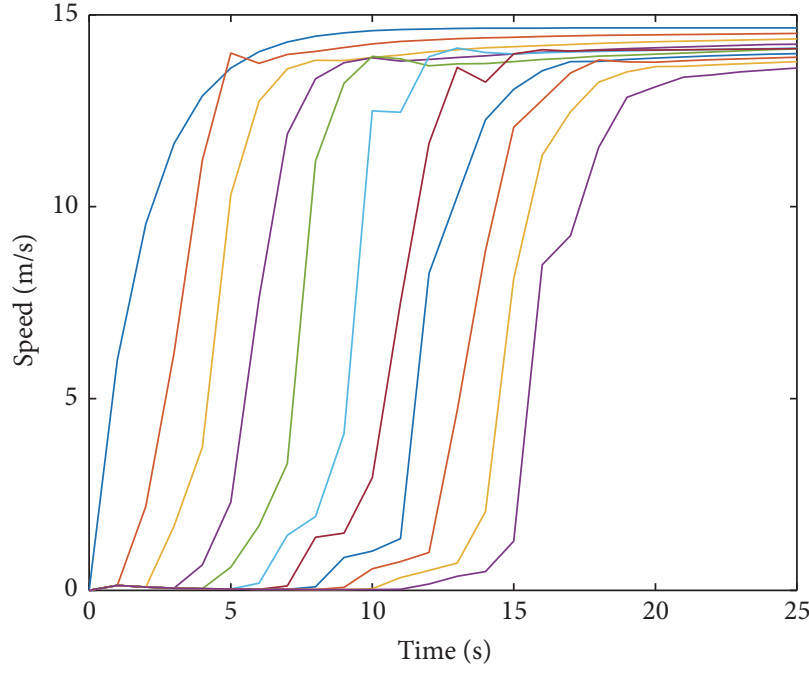

(a)

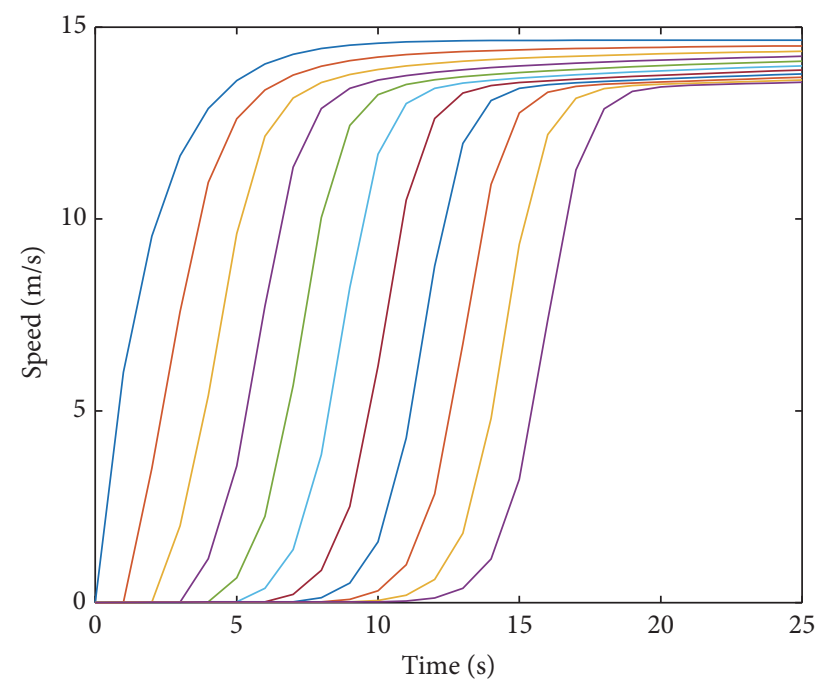

(b)

Figure 6: Velocity evolution curves of the starting process of (a) the proposed model and (b) FVD model $\left(\lambda=0.45, p_{1}=0.2, p_{2}=0.05\right.$, and $\left.p_{3}=-0.2\right)$.

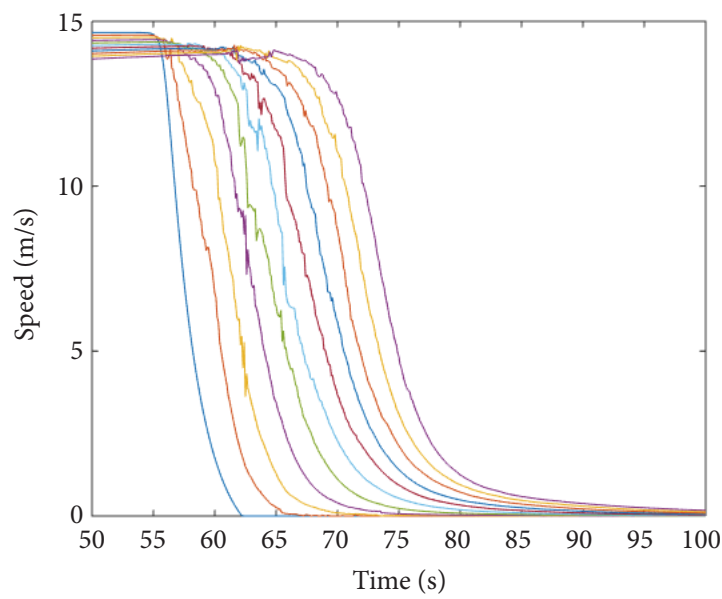

(a)

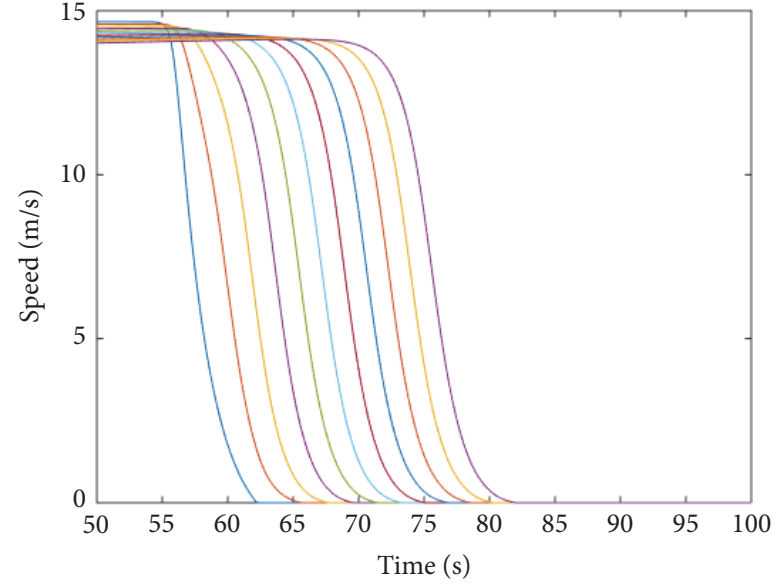

(b)

FIGURE 7: Velocity evolution curves of the braking process of (a) the proposed model and (b) FVD model $\left(\lambda=0.45, p_{1}=0.2, p_{2}=0.05\right.$, and $\left.p_{3}=-0.2\right)$.

For dynamics of a traffic flow, whether disturbances will eventually expand or disappear and whether the traffic flow is stable or not are very important [36]. To check the stability behavior of the proposed model and to further analyze the impact of anticipation characteristic and leading vehicle's acceleration information on the stability of traffic flow, the numerical simulations are carried out under a periodic boundary condition by using the same parameters as in [28]. We set the total cars' number as $N=100$ which are running on a circuit road with length $L=1500 \mathrm{~m}$, the traffic flow is assumed to operate at a uniform spacing $(15 \mathrm{~m})$, and then we apply small perturbations to the head of the vehicle in a steady state and observe the propagation of disturbance over time. The smaller the velocity fluctuation is, the smaller the influence of disturbance on vehicle speed is and the more stable the model is. The initial conditions of the vehicle are as follows:

$$
\begin{aligned}
a & =0.41 \mathrm{~s}^{-1}, \\
\dot{x}_{n}(0) & =V\left(\frac{L}{N}\right), \quad n=1,2, \ldots, N, \\
x_{1}(0) & =1 \mathrm{~m}, \\
x_{n}(0) & =(n-1) \frac{L}{N} \mathrm{~m}, \quad n=2,3, \ldots, N .
\end{aligned}
$$

Figure 8 shows the snapshots of all vehicles' velocities at $t=1000 \mathrm{~s}$ with different improved models based on FVD model. From (a), when the acceleration of leading vehicle is considered, the amplitude fluctuates much more narrowly 


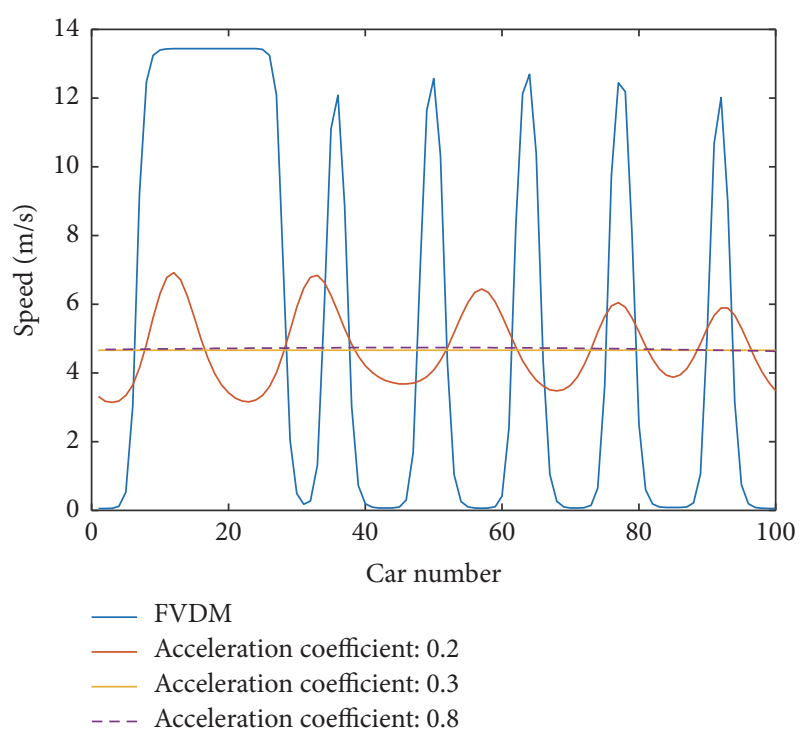

(a)

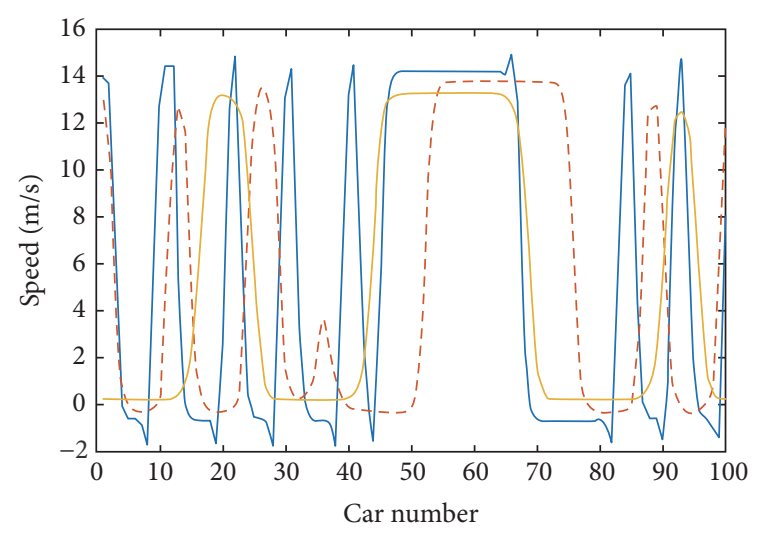

_ Aggressive: 0.3, regular: 0.3, conservative: 0.4

- - - Aggressive: 0.5, regular: 0.3, conservative: 0.2

_ Aggressive: 0.6, regular: 0.2, conservative: 0.2

(c)

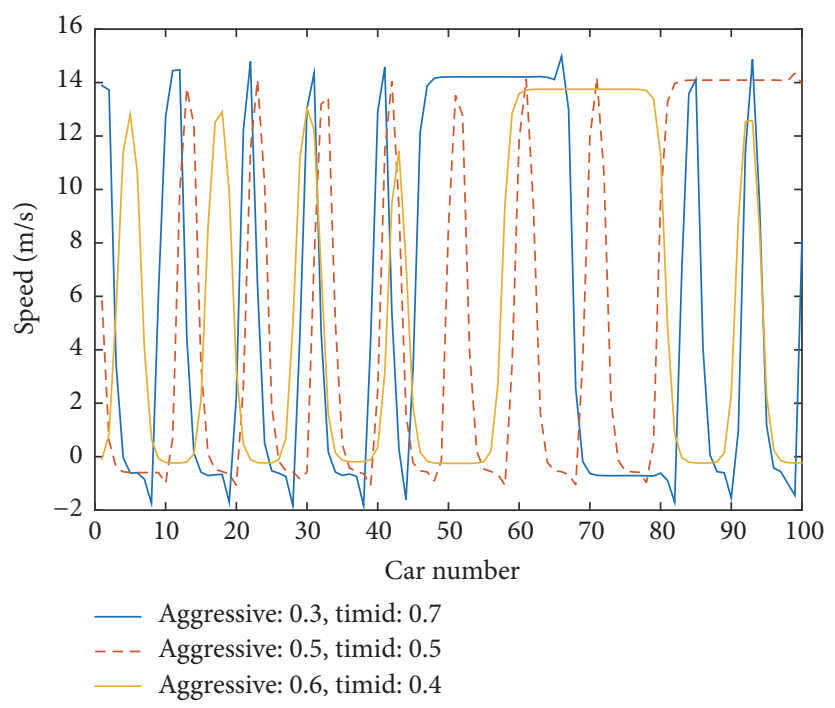

(b)

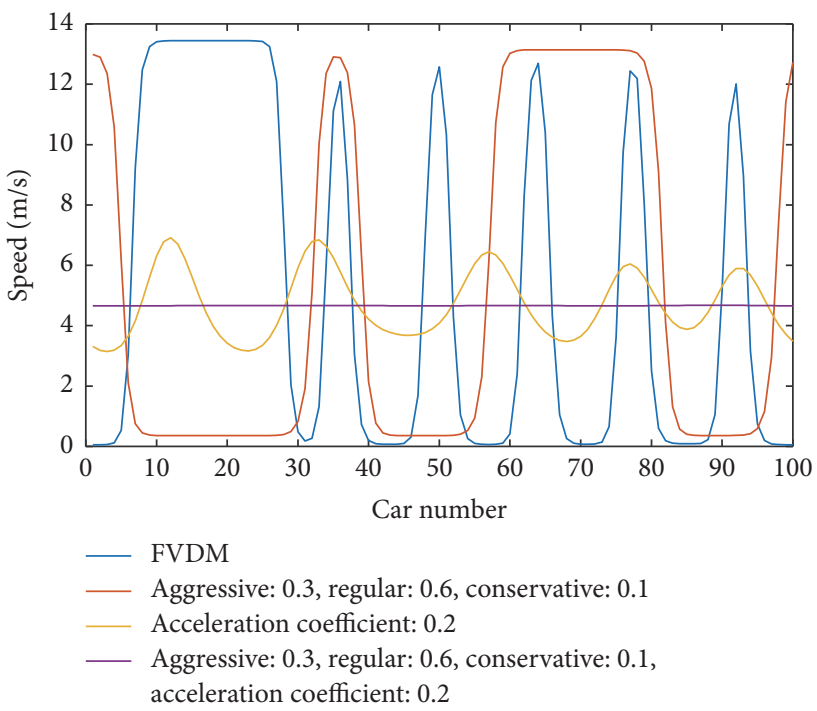

(d)

FIGURE 8: Snapshot of the velocities of all vehicles (a) at different response coefficient to the acceleration of preceding vehicle (b) with two different driving characteristics and (c) with three different driving characteristics and (d) at different parameters in the proposed model when $t=1000 \mathrm{~s}\left(\lambda=0.45, p_{1}=0.2, p_{2}=0.05\right.$, and $\left.p_{3}=-0.2\right)$.

than FVD model (FVDM), which means the stability of traffic flow is improved by introducing the acceleration of leading vehicles. And the stability of traffic flow is obviously improved with the increase of the response coefficient to the acceleration of preceding vehicle. The speed of vehicles is maintained at about $4.8 \mathrm{~m} / \mathrm{s}$ when the response coefficient to the acceleration of preceding vehicle is greater than 0.3. In addition, in order to analyze the effect of different driving styles on traffic flow, first of all, the driving types are divided into two categories according to [28]. We observe the speed fluctuation of 100 vehicles for $1000 \mathrm{~s}$ by giving different weights to the two driving styles of vehicles, and the simulation results are shown in Figure 8(b). Then, the driving types are further divided into three categories and
Figure 8(c) describes the snapshots of all vehicles' velocities at $t=1000 \mathrm{~s}$. It can be seen from Figures 8(b) and 8(c) that when the driving type is divided into 3 classes, the vibration amplitude of vehicles' speed is gentler. It is worth noting that the unrealistic negative velocity, which should be avoided in real traffic, appears in Figures 8(b) and 8(c). And the negative velocity disappears by introducing the acceleration parameters of the leading car. At the same time, from Figure $8(\mathrm{c})$, we can find the amplitude fluctuates more narrowly when the effect of aggressive behaviors is increasing and this is consistent with the experimental results in [28]. From Figure $8(d)$ we can find the stability of traffic flow improves most when the acceleration of leading vehicle and driving characteristics is considered. 


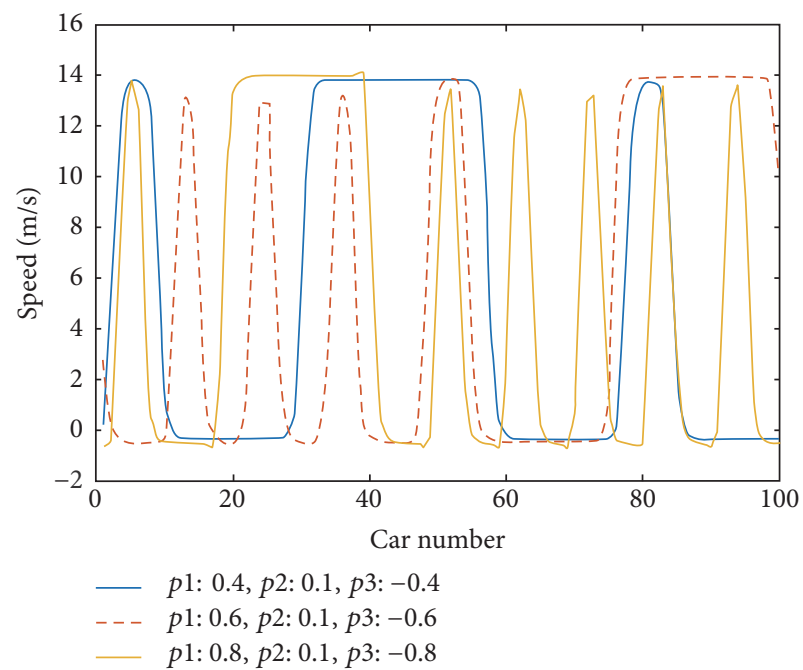

(a) $t=1000 \mathrm{~s}, \lambda=0.45, \alpha=0.3$, and $\beta=0.3$

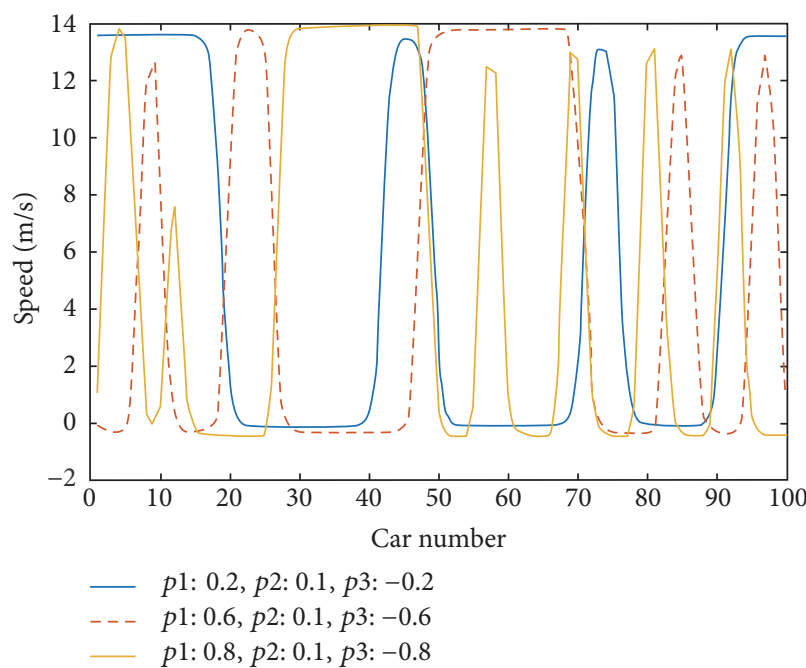

(b) $t=1000 \mathrm{~s}, \lambda=0.45, \alpha=0.2$, and $\beta=0.5$

FIgURE 9: Snapshot of the velocities of all vehicles with different $p_{1}, p_{2}, p_{3}$.

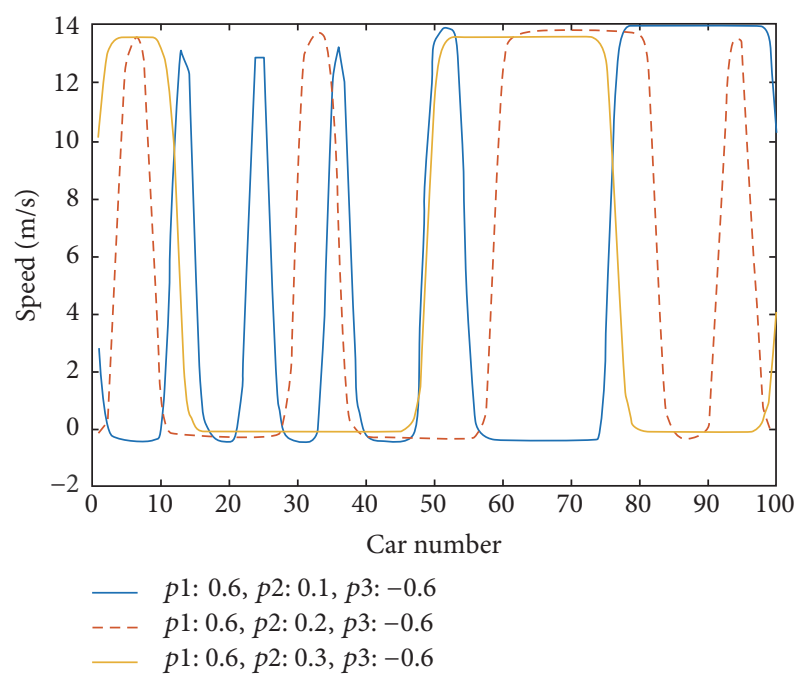

(a) $t=1000 \mathrm{~s}, \lambda=0.45, \alpha=0.3$, and $\beta=0.3$

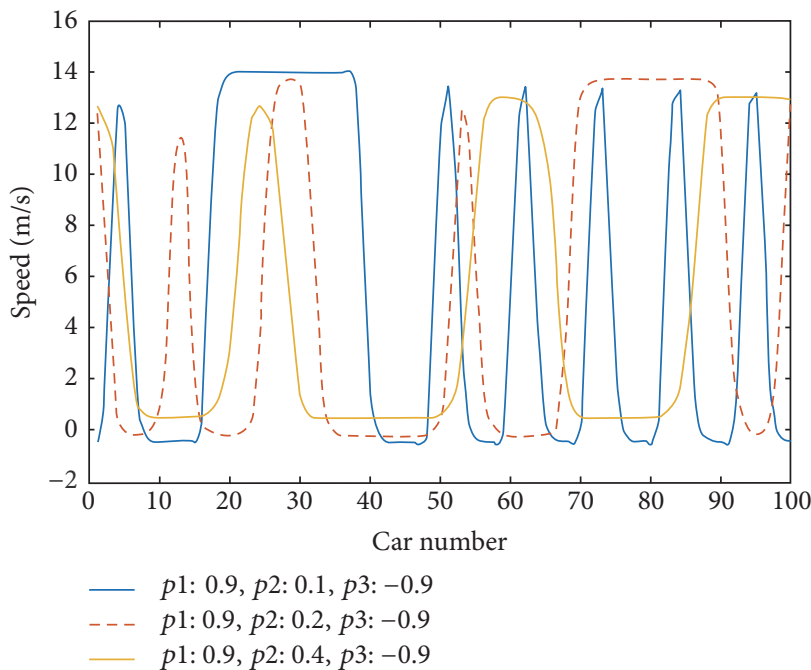

(b) $t=1000 \mathrm{~s}, \lambda=0.45, \alpha=0.2$, and $\beta=0.5$

Figure 10: Snapshot of the velocities of all vehicles with different $p_{1}, p_{2}, p_{3}$.

In Figures 9 and 10, the car's velocity is denoted with different $p_{1}, p_{2}, p_{3}$ corresponding to $\alpha=0.3, \beta=0.3$ and $\alpha=0.2, \beta=0.5$, respectively. Figures 9 and 10, respectively, demonstrate that the traffic flow will be much more stable with the decrease of delay time of aggressive $\left(p_{1}\right)$ and conservative $\left(p_{3}\right)$ type of driving styles and with the increase of delay time of regular $\left(p_{2}\right)$ type of driving style.

Figure 11 shows the velocity-headway trajectory of FVD model (FVDM), (a) a model considering the acceleration of leading vehicle on the basis of FVD model, (b) a model with different driving characteristics introduced, and (c) the proposed model. After sufficiently long time, the closed trajectories called hysteresis loops are explicitly observed. It can be clearly observed from (a) that the negative velocity appearing in hysteresis loop of FVD model disappears when the acceleration of leading vehicle is considered. Besides that, as the response coefficient to the acceleration of preceding vehicle increases, the area of hysteresis loop becomes progressively smaller, which denotes that the introduction of preceding vehicle's acceleration plays a positive role in the stability of traffic flow. From (b), the sizes of hysteresis loop are enlarged with the increase of conservative characteristics which are in accordance with above simulation results. Similarly, the negative velocity in hysteresis loop disappears with the increase of regular and aggressive characteristics. It is clearly illustrated in (c) that comprehensive consideration of preceding vehicle's acceleration and driving characteristics can greatly enhance traffic stability. 


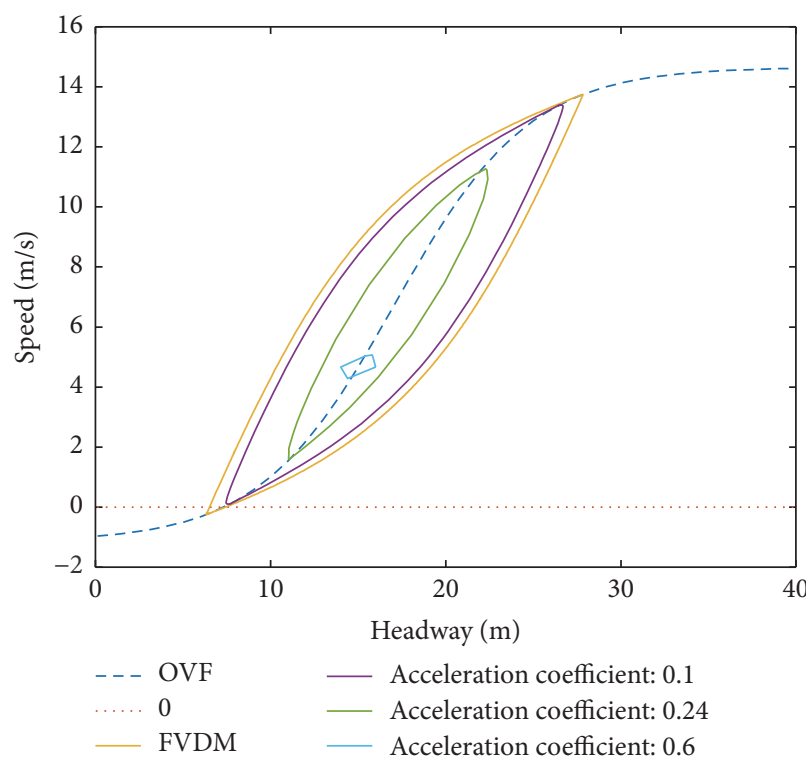

(a)

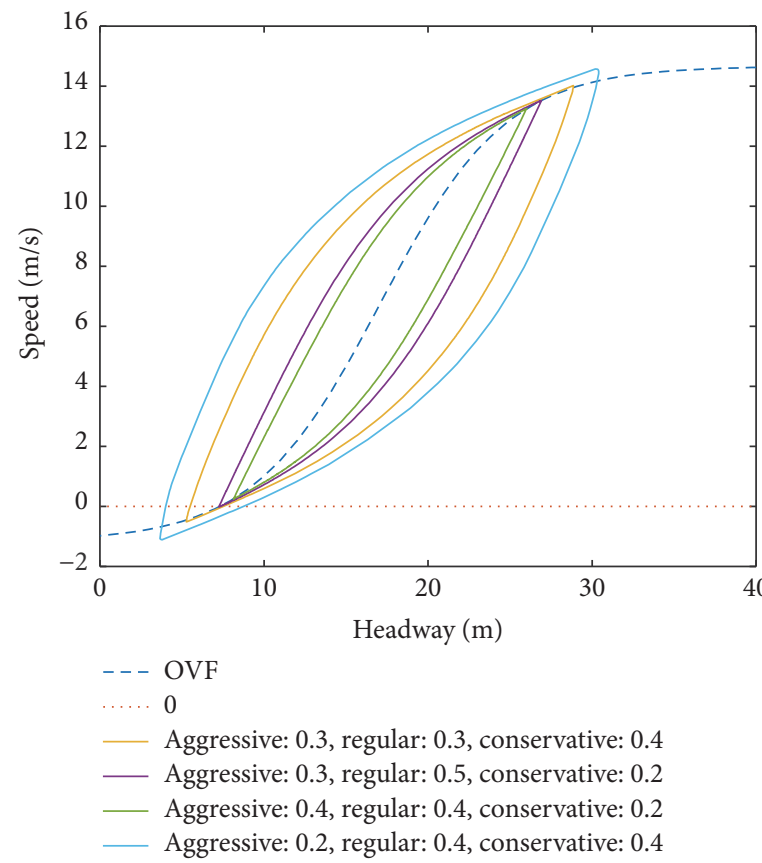

(b)

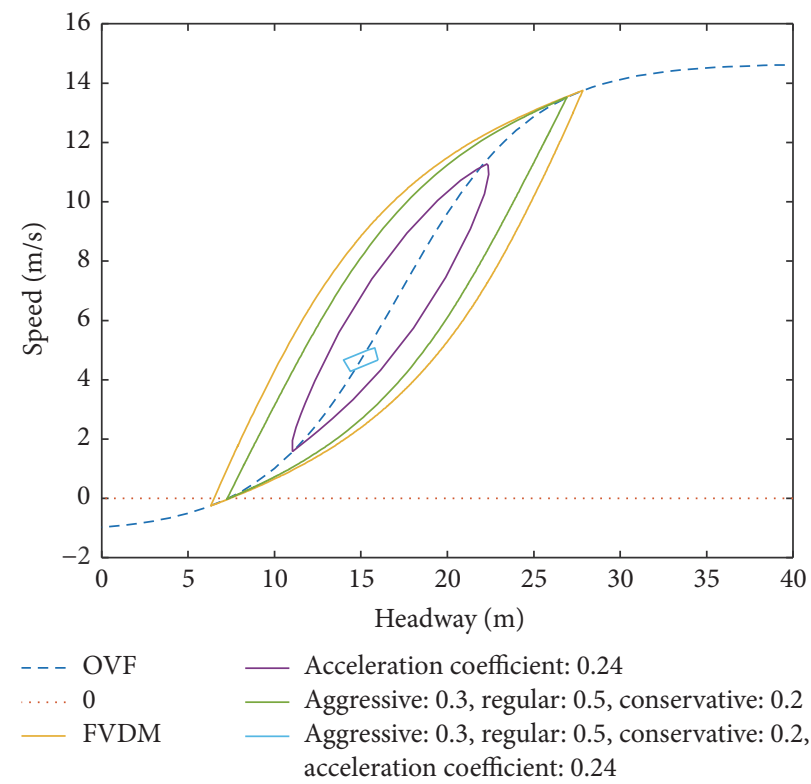

(c)

FIGURE 11: The loops for (a) a model considering the acceleration of leading vehicle and (b) a model considering driving characteristics and (c) the proposed model $\left(\lambda=0.45, p_{1}=0.3, p_{2}=0.1\right.$, and $\left.p_{3}=-0.3\right)$. OVF is short for optimal velocity function and FVDM is short for full velocity difference model.

\section{Conclusions}

This paper proposes a new model based on FVD model by considering the acceleration of preceding vehicle and the division of driving types derived from real traffic data. A clustering algorithm is adopted to divide the driving types; then an analysis on the clustering results is given, which demonstrates that different drivers have different driving styles. According to the three driving styles obtained by clustering, a new model is proposed. And the linear stable condition of the proposed model is obtained by making use of linear stability analysis. Then the effect of preceding vehicle's acceleration and driving characteristics on the stability criterion is further researched. The results of numerical simulation prove that the introduction of preceding vehicle's acceleration can avoid the negative velocity appearing in FVD model. We can find that the stability of the models with three types of driving is better than that of the one with the 
two driving styles. And more aggressive and regular effect can avoid the disadvantage of negative velocity and negative headway occurring with conservative effect dominant in optimal velocity. The aggressive and regular behavior play a positive role in the stabilization of traffic flow while the conservative behavior deteriorates traffic stability. Thus, this new model is more stable than the classic FVD model and is more realistic in describing real traffic flow than FVD model which supposes that all drivers are with same neutral attribute.

In the future work, a quantitative study can be made on the response time of different driving characteristics based on more field data.

\section{Conflicts of Interest}

The authors declare that the mentioned received funding in Acknowledgments does not lead to any conflicts of interest regarding the publication of this manuscript. And there are no extra conflicts of interest in this manuscript.

\section{Acknowledgments}

This work is supported by National Natural Science Foundation of China (Grant no. 61300065), Beijing Natural Science Foundation (Grant no. 4142010), Beijing Municipal Science \& Technology Project (Grant nos. Z151100002115040, Z171100000517003, Z171100000517004, Z161100001116072, and Z171100004417023), and Project of Beijing Municipal Education Commission (Grant no. KM201610005033).

\section{References}

[1] J. D. Wang and J. L. Zhang, "Stability analysis and simulation research of improved full velocity difference model," Computer Engineering and Applications, vol. 52, no. 7, pp. 24-27, 2016.

[2] L. A. Pipes, "An operational analysis of traffic dynamics," Journal of Applied Physics, vol. 24, no. 3, pp. 274-281, 1953.

[3] M. Bando, K. Hasebe, A. Nakayama, A. Shibata, and Y. Sugiyama, "Dynamical model of traffic congestion and numerical simulation," Physical Review E, vol. 51, no. 2, pp. 1035-1042, 1995.

[4] D. Helbing and B. Tilch, "Generalized force model of traffic dynamics," Physical Review E, vol. 58, no. 1, pp. 133-138, 1998.

[5] R. Jiang, Q. S. Wu, and Z. J. Zhu, "Full velocity difference model for a car-following theory," Physical Review E, vol. 64, no. 2, Article ID 017101, 2001.

[6] T. Q. Tang, J. G. Li, H. J. Huang, and X. B. Yang, "A carfollowing model with real-time road conditions and numerical tests," Measurement, vol. 48, no. 1, pp. 63-76, 2014.

[7] T. Tang, W. Shi, H. Shang, and Y. Wang, "A new car-following model with consideration of inter-vehicle communication," Nonlinear Dynamics, vol. 76, no. 4, pp. 2017-2023, 2014.

[8] H. X. Gong, H. C. Liu, and B.-H. Wang, "An asymmetric full velocity difference car-following model," Physica A, vol. 387, no. 11, pp. 2595-2602, 2008.

[9] L.-J. Zheng, C. Tian, D.-H. Sun, and W.-N. Liu, "A new carfollowing model with consideration of anticipation driving behavior," Nonlinear Dynamics. An International Journal of
Nonlinear Dynamics and Chaos in Engineering Systems, vol. 70, no. 2, pp. 1205-1211, 2012.

[10] H. X. Ge, R. J. Cheng, and Z. P. Li, “Two velocity difference model for a car following theory," Physica A: Statistical Mechanics and Its Applications, vol. 387, no. 21, pp. 5239-5245, 2008.

[11] H. X. Ge, S. Q. Dai, L. Y. Dong, and Y. Xue, "Stabilization effect of traffic flow in an extended car-following model based on an intelligent transportation system application," Physical Review E: Statistical, Nonlinear, and Soft Matter Physics, vol. 70, no. 6, Article ID 066134, 2004.

[12] G. H. Peng, X. H. Cai, and C. Q. Liu, "Optimal velocity difference model for a car-following theory," Fuel and Energy Abstracts, vol. 375, no. 45, pp. 3973-3977, 2011.

[13] G. H. Peng and D. H. Sun, "A dynamical model of car-following with the consideration of the multiple information of preceding cars," Physics Letters, Section A: General, Atomic and Solid State Physics, vol. 374, no. 15-16, pp. 1694-1698, 2010.

[14] D. Sun, X. Liao, and G. Peng, "Effect of looking backward on traffic flow in an extended multiple car-following model," Physica A: Statistical Mechanics and its Applications, vol. 390, no. 4, pp. 631-635, 2011.

[15] D. H. Sun, M. Zhang, and T. Chuan, "Multiple optimal current difference effect in the lattice traffic flow model," Modern Physics Letters B, vol. 28, no. 11, pp. 610-621, 2014.

[16] T. Wang, Z. Y. Gao, and X. M. Zhao, "Multiple velocity difference model and its stability analysis," Acta Physica Sinica, vol. 55, no. 2, pp. 634-640, 2006.

[17] H. X. Ge, P. J. Zheng, and W. Wang, “The car following model considering traffic jerk," Physica A Statistical Mechanics and Its Applications, vol. 433, pp. 274-278, 2015.

[18] H. Song, H. Ge, F. Chen, and R. Cheng, "TDGL and mKdV equations for car-following model considering traffic jerk and velocity difference," Nonlinear Dynamics, vol. 87, no. 3, pp. 18091817, 2017.

[19] T. Q. Tang, C. Y. Li, and H. J. Huang, "A new car-following model with the consideration of the driver's forecast effect," Physics Letters A, vol. 374, no. 38, pp. 3951-3956, 2010.

[20] G. H. Peng, "A study of wide moving jams in a new lattice model of traffic flow with the consideration of the driver anticipation effect and numerical simulation," Physica A Statistical Mechanics and Its Applications, vol. 391, no. 23, pp. 5971-5977, 2012.

[21] T. Q. Tang, H. J. Huang, and H. Y. Shang, "A new macro model for traffic flow with the consideration of the driver's forecast effect," Physics Letters, Section A: General, Atomic and Solid State Physics, vol. 374, no. 15-16, pp. 1668-1672, 2010.

[22] T. Kim, D. Lovell, H. Kim, and C. Oh, "Empirical results of effects of various causal factors on car-following behavior," Transportation Research Record: Journal of the Transportation Research Board, vol. 2188, pp. 174-186, 2010.

[23] S. Ossen and S. Hoogendoorn, "Car-following behavior analysis from microscopic trajectory data," Transportation Research Record: Journal of the Transportation Research Board, vol. 1934, pp. 13-21, 2005.

[24] M. Treiber, A. Kesting, and D. Helbing, "inaccuracies and anticipation in microscopic traffic models," Physica A Statistical Mechanics Its Applications, vol. 360, no. 1, pp. 71-88, 2006.

[25] S. Sharma, "Modeling and analyses of driver's characteristics in a traffic system with passing," Nonlinear Dynamics, vol. 86, no. 3, pp. 2093-2104, 2016.

[26] H. Wen, Y. Rong, C. Zeng, and W. Qi, “The effect of driver's characteristics on the stability of traffic flow under honk 
environment," Nonlinear Dynamics, vol. 84, no. 3, pp. 1517-1528, 2016.

[27] A. K. Gupta and P. Redhu, "Analyses of the driver's anticipation effect in a new lattice hydrodynamic traffic flow model with passing," Nonlinear Dynamics. An International Journal of Nonlinear Dynamics and Chaos in Engineering Systems, vol. 76, no. 2, pp. 1001-1011, 2014.

[28] G. H. Peng, H. D. He, and W. Z. Lu, "A new car-following model with the consideration of incorporating timid and aggressive driving behaviors," Physica A Statistical Mechanics and Its Applications, vol. 442, pp. 197-202, 2016.

[29] B. Sultan, M. Brackstone, and M. McDonald, "Drivers' use of deceleration and acceleration information in car-following process," Transportation Research Record: Journal of the Transportation Research Board, vol. 1883, pp. 31-39, 2004.

[30] S. Juma, Z. Muda, and W. Yassin, "Reducing false alarm using hybrid intrusion detection based on $\mathrm{x}$-means clustering and random forest classification," Journal of Theoretical Applied Information Technology, 2014.

[31] NGSIM: Next Generation Simulation, FHWA, U.S. Department of Transportation, http://www.ngsim.fhwa.dot.gov.

[32] C. Thiemann, M. Treiber, and A. Kesting, "Estimating acceleration and lane-changing dynamics from next generation simulation trajectory data," Transportation Research Record, vol. 2088, pp. 90-101, 2008.

[33] H. Deng and H. Zhang, "Driver anticipation in car following," Transportation Research Record: Journal of the Transportation Research Board, vol. 2316, pp. 31-37, 2012.

[34] H. U. Zhiying, "Numerical simulation of car-following model considering preceding vehicle's acceleration," Computer Engineering and Applications, vol. 51, no. 11, pp. 47-49, 2015.

[35] G. G. Schultz, "Analysis of distribution and calibration of carfollowing sensitivity parameters in microscopic traffic simulation models," Transportation Research Record: Journal of the Transportation Research Board, vol. 1876, pp. 41-51, 2004.

[36] L. S. J. Ossen, Longitudinal Driving Behavior: Theory and Empirics, 2008, Drivers. 


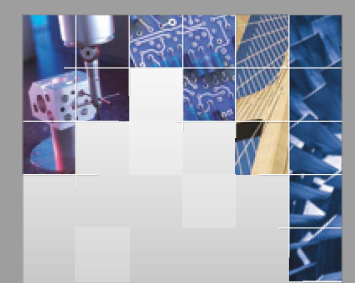

\section{Enfincering}
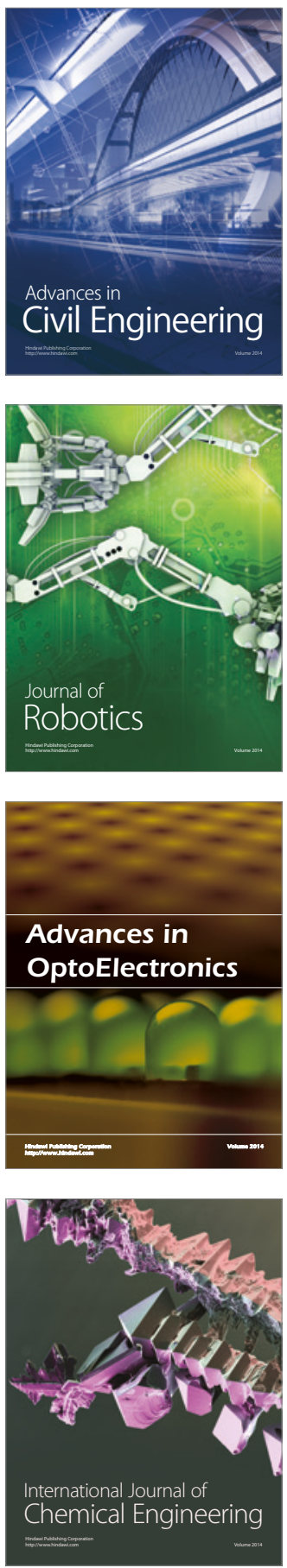

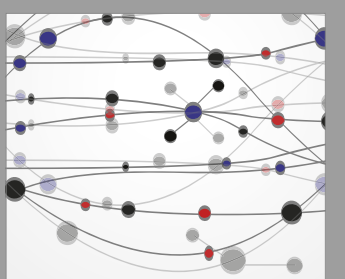

The Scientific World Journal

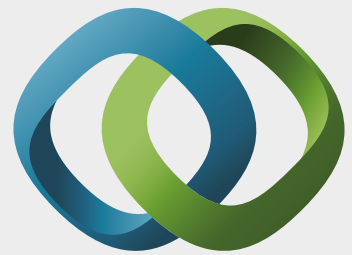

\section{Hindawi}

Submit your manuscripts at

https://www.hindawi.com
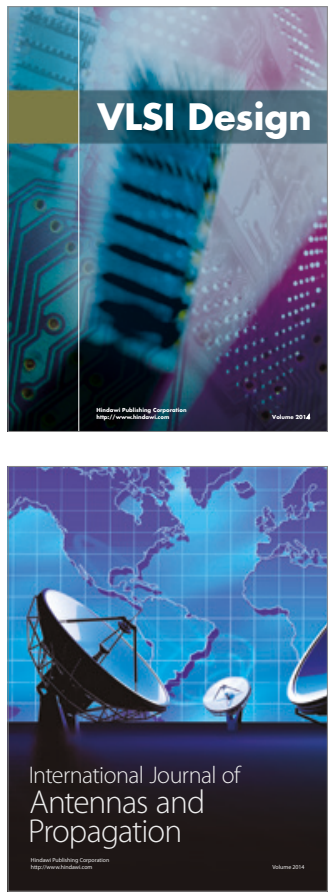

\section{Rotating}

Machinery
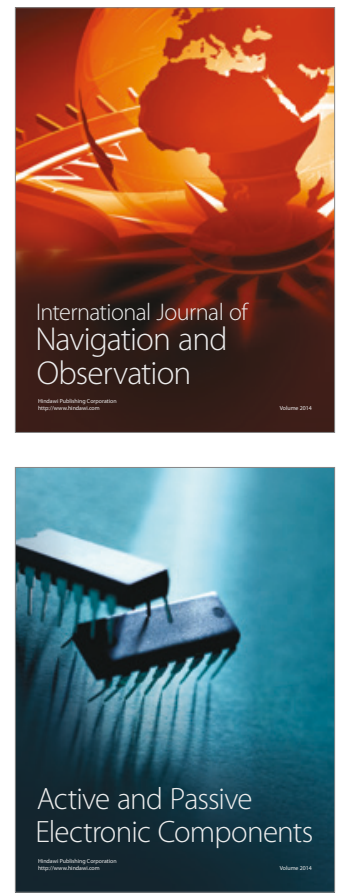
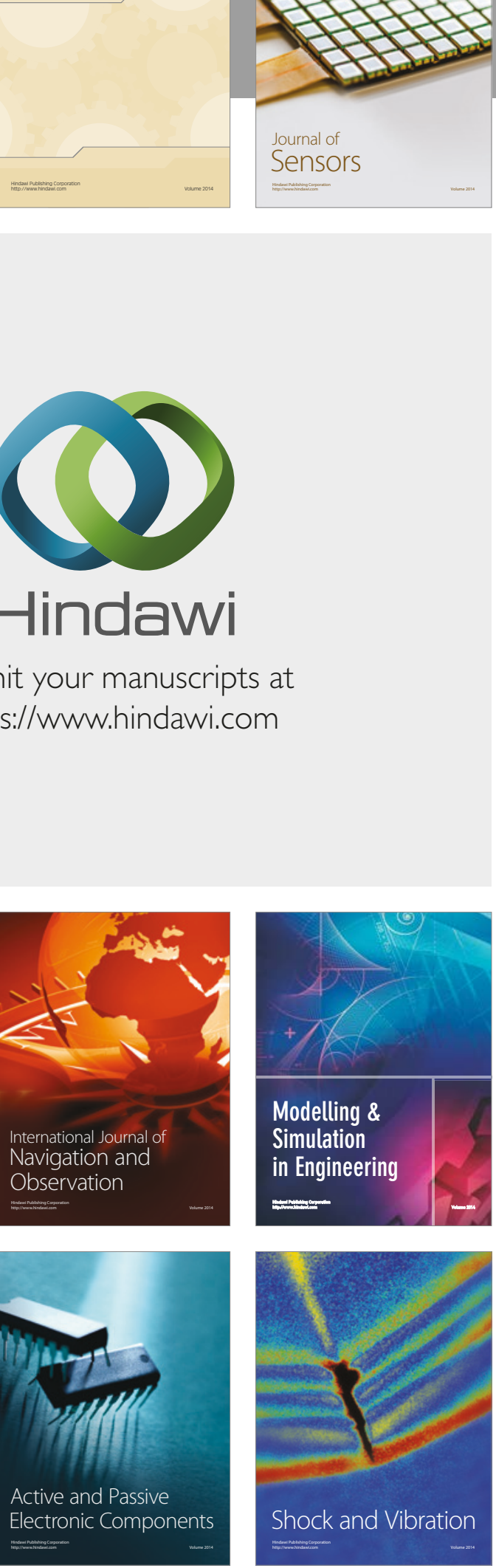
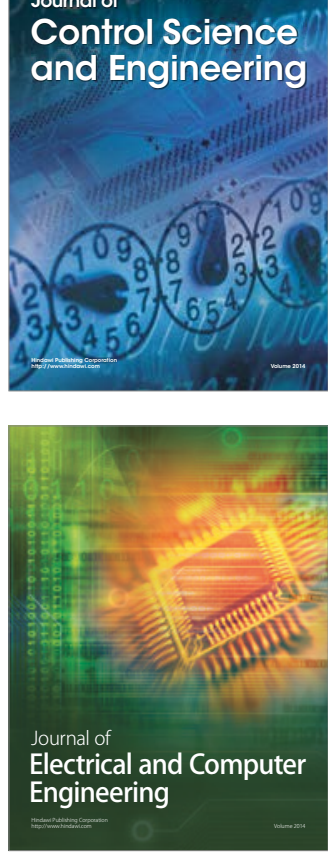

Distributed

Journal of

Control Science

and Engineering
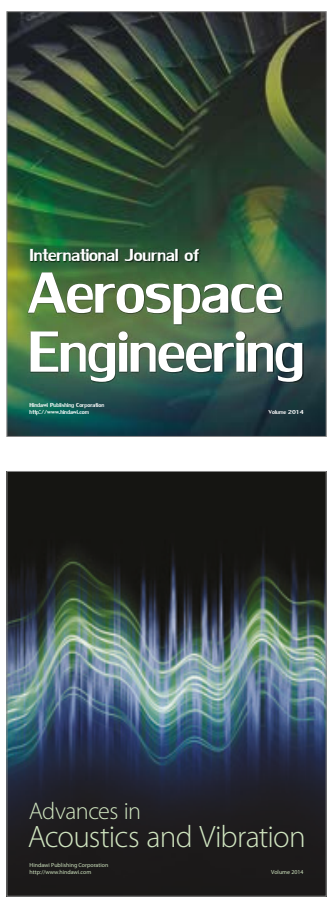

Sensor Networks 